\title{
Genome-wide association study reveals novel genetic variants associated with HIV-1C infection in Botswana population
}

\author{
Andrey K. Shevchenko \\ Theodosius Dobzhansky Center for \\ Genome Bioinformatics \\ St.-Petersburg State University \\ St-Petersburg, Russia \\ ORCID: 0000-0002-7332-5661
}

\author{
Sergey V. Malov \\ Theodosius Dobzhansky Center for \\ Genome Bioinformatics \\ St.-Petersburg State University \\ St-Petersburg, Russia \\ ORCID: 0000-0003-0093-6506
}

\author{
Alexey Antonik \\ Theodosius Dobzhansky Center for \\ Genome Bioinformatics \\ St.-Petersburg State University \\ St-Petersburg, Russia \\ ORCID: 0000-0003-4341-1291
}

\begin{abstract}
Genome wide association studies (GWAS) allow to identify common variants associated with the trait in question. In order to efficiently search for the genetic associations we have previously developed Genome-Wide Association Tracks Chromosome Highway (GWATCH). The broad goal of the Botswana GWAS project is to identify genetic determinants of susceptibility and resistance to infection by HIV-1 subtype $\mathrm{C}$ among people severely affected by HIV/AIDS in Botswana. By conducting GWAS analysis on HIV1C case/control dataset consisting of 762 Tswana people (combined from two partly overlapping datasets of 809 microarray and 362 WGS samples), we found several gene regions slightly below significance level.
\end{abstract}

Keywords - GWAS, GWATCH, HIV-1, AIDS, Botswana

\section{Introduction}

HIV-infection is a deadly chronic disease spreading across all continents. There are 37.9 million $[32.7$ million- 44.0 million] people in the world living with HIV; 35.4 million [25.0 million-49.9 million] people who died from AIDSrelated conditions since the onset of the epidemic several decades ago with the estimated 770000 [570 000-1.1 million] who died in 2018 (UNAIDS, 2019). Despite the efforts and resources dedicated to the fight against the HIV epidemics for more than three decades, currently no effective vaccine or cure exists.

According to UNAIDS, Botswana falls into the top 3 countries with the highest HIV prevalence in the world with an adult aged 15 to 49 HIV prevalence rate of 20.3 [17.3 21.8] (UNAIDS, 2019). Moreover, HIV-1 subtype C (widespread in Botswana), which accounts for over half of HIV-1 infections worldwide, is understudied, because most current studies focus on other HIV subtypes more prevalent in Europe and the USA. We wanted to fill this gap and to search for genetic factors associated with $\mathrm{HIV}-1$ subtype $\mathrm{C}$ in Botswana population.

\section{Methods}

The individuals used in this study originated from two primary populations that have been described in detail. One was a population of pregnant women (436) involved in a maternal transmission study; the other (373) was a population enrolled to identify acute or early infections. This data were obtained in collaboration with Harvard T.H. Chan School of Public Health AIDS Initiative (Boston, USA) and Botswana Harvard AIDS Institute (Gaborone, Botswana). These samples were genotyped using two technologies: 809 samples have microarray-based genotypes (this dataset is called $\mathrm{BH}$ IM), and 364 samples (335 of which are overlapping with these 809) have WGS-based genotypes. The resulting genotype data was used for the association tests, performed using PLINK logistic regression. These results were loaded into the GWATCH tool [Svitin et al, 2014; http://genwatch.org/] for further analysis and visualization.

\section{Results}

First, we were able to replicate known HIV-associated loci from [O'Brien 2013] (NCOR2, TRIM5, CXCL12 and several HLA genes). Next, we investigated the newly discovered loci associated with HIV-1 subtype C. Many of the top associated genes are known to play a role in pathways that may be important in HIV infection process, such as L3MBTL3, NEO1, CD79B, AP3B1, PTPRA and others. One of the identified loci (close to HCG22 gene) was also replicated in another dataset [Xie et al. 2016].

\section{Conclusions}

This work presents the first step towards identification of genetic variants associated with infection by HIV-1 subtype C in Botswana population. We have replicated several loci that have shown association with HIV in previous studies. In addition, we have identified several novel putative associated loci in such genes as NEO1, CD79B, L3MBTL3, AP3B1, PTPRA.

\section{ACKNOWLEDGMENT}

This research was supported in part by St.-Petersburg State University (grant No. 1.52.1647.2016)

\section{REFERENCES}

[1] Svitin A. et al. 2014. GWATCH: a web platform for automated gene association discovery analysis. GigaScience.

[2] Xie W. et al. 2016. Genome-Wide Analyses Reveal Gene Influence on HIV Disease progression and HIV-1C Acquisition in Southern Africa. AIDS Research and Human Retroviruses.

[3] O'Brien SJ, Hendrickson SL. Host genomic influences on HIV/AIDS. Genome Biol. 2013 Jan 31;14(1):201. doi:10.1186/gb-2013-14-1-201 